\title{
A IMPORTÂNCIA DO CONHECIMENTO GEOTECNOLÓGICO NO CURSO TÉCNICO EM RECURSOS PESQUEIROS DO IFRN
}

\author{
N. F. C. França; P. V. N Araújo; G. G. Albino \\ Instituto Federal de Educação, Ciência e Tecnologia do Rio Grande do Norte (IFRN) \\ niferiuz_franca@hotmail.com; paulo.araujo@ifrn.edu.br; giovana.albino@ifrn.edu.br \\ 10.15628/rbept.2017.5111
}

Artigo submetido em set/2016 e aceito em mar/2017

\section{RESUMO}

O mundo globalizado contempla um grande período de avanço e expansão tecnológica, aumentando a cada minuto seu vasto número de usuários. Vivemos uma contínua fase de atualização dos conhecimentos diante das mudanças e evoluções dos mais diversos tipos de tecnologia. Dentre as quais, destacamos as geotecnologias como protagonistas do nosso estudo. 0 crescente interesse geotecnológico no Brasil vem despontando significativamente nos últimos anos e as mais diversas áreas de conhecimento vêm usufruindo dos benefícios gerados pela utilização dessa tecnologia, cada vez mais presente em nosso cotidiano. O presente trabalho tem como objetivo propor uma revisão curricular no Curso Técnico em Recursos Pesqueiros do Instituto Federal de Educação, Ciência e Tecnologia do Rio Grande do Norte (IFRN), de modo a proporcionar a aquisição de um novo componente curricular que trabalhe com ferramentas geotecnológicas aplicadas à área de atuação de Recursos Pesqueiros. Nossa metodologia contemplou uma pesquisa bibliográfica e utilização de um questionário com o corpo docente do instituto em foco, que abordava questões sobre a importância do ensino de geoprocessamento em Recursos Pesqueiros. Como resultados alcançados, confirmamos a importância de uma revisão curricular no curso técnico em questão, prevendo a criação desse novo componente curricular, a fim de promover um diferencial na vida acadêmica e profissional dos futuros técnicos em Recursos Pesqueiros. Com isto, seria mantida, assim, a tradição da instituição pesquisada em formar profissionais de referência para o competitivo mercado de trabalho.

Palavras-Chaves: Educação Profissional; Currículo; Recursos Pesqueiros; Geotecnologia. 


\section{ABSTRACT}

The globalized world has been watching a great period of technological development and expansion that increases its number of users each minute. We live in a continuous phase of knowledge update facing the changes and evolutions of the most different types of technology. Among those, we emphasize the geotechnologies as leading figures of our study. The growing geotechnological interest in Brazil has been emerging significantly in the last years, and the most diverse areas of knowledge have been profiting from the benefits generated from the use of this technology that is more and more constant in our daily life. This work aims to propose a curricular review of the Fishery Resources Technician Course from the Federal Institute of Education, Science and Technology of Rio Grande do Norte (IFRN) in order to provide the acquisition of a new curricular component that deals with geotechnological tools applied to the Fishery Resources area of work. Our methodology consisted of a bibliographical research, and the use of a questionnaire directed to the teaching staff from the given institution with questions on the importance of teaching geoprocessing in the Fishery Resources course. As regards the results achieved, we confirm the importance of a curricular review in the technical course in question, anticipating the creation of this new curricular component for the sake of promoting a differential in the academic and professional live of future Fishery Resources technicians. As a result, the studied institution would thus maintain its tradition of training well-recognized professionals for the ever-competitive job market.

KEYWORDS: Professional Education; Curriculum; Fishery Resources; Geotechnology. 


\section{INTRODUÇÃO}

O Instituto Federal de Educação, Ciência e Tecnologia do Rio Grande do Norte (IFRN), oferta, no Campus Macau, o único Curso Técnico em Recursos Pesqueiros do Estado. Embora apresente toda uma trajetória de conquistas, com um ensino e um corpo docente que primam pela qualidade, o respectivo curso técnico possui uma carência em sua matriz curricular que contemple o estudo de geotecnologias aplicadas à área de recursos pesqueiros, ainda que estas sejam, hoje, uma grande ferramenta aliada à gestão ambiental e pesqueira inclusive para abordagens práticas voltadas para a conscientização e a importância ambientais.

Sabendo da necessidade de adequar-se à realidade atual de grande avanço tecnológico e embasado em pesquisas realizadas nas áreas mais desenvolvidas do globo, que comprovam a relevância da utilização da geotecnologia em diversas áreas de conhecimento, o presente trabalho promove uma discussão sobre o currículo atual do Curso Técnico em Recursos Pesqueiros, defendendo a proposta de uma revisão curricular que contemple o acesso ao ensino da geotecnologia como uma aliada à área de atuação dos Recursos Pesqueiros.

O objetivo geral do trabalho consiste em propor uma revisão no currículo do referido curso técnico que proporcione a aquisição de um novo componente curricular voltado à utilização de ferramentas geotecnológicas aplicadas à área de atuação de Recursos Pesqueiros.

\section{O QUE SÃO AS GEOTECNOLOGIAS? E QUAIS AS SUAS FUNCIONALIDADES?}

O mundo globalizado contempla um grande período de avanço e expansão tecnológica, aumentando o seu número de usuários. Vivemos uma contínua fase de atualização dos conhecimentos diante das mudanças e evoluções de diversos tipos de tecnologias. Dentre estas se apresentam as geotecnologias, que atualmente disputam o ranking dos mercados emergentes em nível mundial, ao lado da nanotecnologia e da biotecnologia (SONSOLO e BACK, 2008).

A introdução do geoprocessamento no Brasil teve início em meados dos anos 80, na Universidade Federal do Rio de Janeiro (UFRJ), em trabalhos de divulgação e capacitação realizados pelo professor Jorge Xavier da Silva (CÂMARA et. al. apud SANSOLO, 2008, p. 3). 0 crescente interesse pelas geotecnologias no Brasil vem despontando significativamente nos últimos anos. As mais diversificadas áreas de conhecimento vêm usufruindo dos benefícios gerados pela utilização dessas tecnologias, que estão cada vez mais presentes em nosso cotidiano, como por exemplo: em aparelhos celulares; rastreadores veiculares; navegadores veiculares; sites de mapas, entre outros. De acordo com o portal nacional especializado em geotecnologias, o FatorGis, "As geotecnologias são um conjunto de tecnologias para a coleta, processamento, análise e disponibilização de informações de referência geográfica. [...] são compostas por soluções em hardware, software e peopleware, que juntas se constituem em poderosas ferramentas para tomada de decisão" (SONSOLO e BACK, 2008, p. 3).

As geotecnologias comportam uma série de tecnologias. Em meio a elas estão inseridos os Sistemas de Informação Geográfica (SIG) ou do inglês Geographic Information System (GIS), termo muito utilizado por estudiosos da área, de forma global. Segundo Sonsolo e Back (2008, p. 3), os SIG são "[...] sistemas de informações construídos especialmente para armazenar, analisar e manipular dados geográficos, ou seja, dados que representam objetos e fenômenos em que a localização geográfica é uma característica inerente e indispensável para tratá-los". 
Os SIG são essencialmente softwares que reúnem recursos gráficos e de bancos de dados relacionados. Os dados possuem referências geográficas, sendo posicionados por suas coordenadas. Através da utilização de dados espaciais (cartográficos) e alfanuméricos tabulares, esses sistemas permitem a obtenção de novas informações. Eles têm como característica o trabalho com diversos planos de informações, permitindo a utilização de modelos matemáticos para os processos de análise das informações de planos sobrepostos. Assim, um SIG pode ser composto por quatro partes básicas: o computador e demais equipamentos (hardware) que podem ser utilizados para aquisição, armazenamento e processamento de dados; programas específicos (software); dados (banco de dados georreferenciados/tabelas etc.) e o próprio usuário a interagir com todos esses elementos, denominado por peopleware.

No entender de Câmara (apud SANSOLO, 2008, p. 4), um SIG tem os seguintes componentes: Interface com o usuário; entrada e integração de dados; funções de processamento gráfico e de imagem; visualização e plotagem; e banco de dados geográficos.

Seguindo essa composição, a utilização desses sistemas deve, com toda certeza, auxiliar na construção do conhecimento e a solução de problemas de ordem espacial, uma vez que os SIG possibilitam o armazenamento, manipulação e análise de dados, que podem gerar resultados em forma de produtos cartográficos (mapas), tabelas e gráficos. O emprego do SIG, além de trabalhar com feições do mundo real, permite que sejam gerados cenários de representação territorial futura (SONSOLO e BACK, 2008, p. 4).

“Pode-se dizer então que, de maneira geral, os SIG podem ser utilizados quando nos depararmos com a palavra 'onde' em nossas questões e problemas que necessitam de solução por meio de um sistema informatizado" (CÂMARA et. al. apud SANSOLO e BACK, 2008, p. 4).

Nessa perspectiva, a tecnologia de geoprocessamento apresenta-se, em meio ao estudo dos recursos pesqueiros, como uma aposta significativa de apoio às tomadas de decisões em termos de aplicações de ampla escala, fazendo sempre o uso de sistemas integrados de geoinformação com aplicações específicas de dados pesqueiros. De acordo com Fisher e Rahel (apud SOUSA, 2009, p. 4430), "os SIG são ambientes computacionais habilitados para descrever tais complexidades e podem aumentar a capacidade de visualizar e analisar tais processos em diferentes tipologias espaciais e temporais pesqueiras".

No entender de Babcock et al. (apud SOUSA, 2009, p. 4430), os mesmos podem ser aplicados ao monitoramento e manejo de populações marinhas, algo crescente nos últimos anos. Nesse sentido, "algumas aplicabilidades de SIG em pesca têm sido direcionadas para elaboração de produtos temáticos como mapeamento da distribuição de estoques, modelagem espacial e determinação de habitats essenciais de peixes" (FISHER e TOEPFER apud SOUSA, 2009, p. 4430).

A tabela 1 apresenta a síntese e algumas das principais linhas de estudo da geoinformação com enfoque nos recursos pesqueiros. 


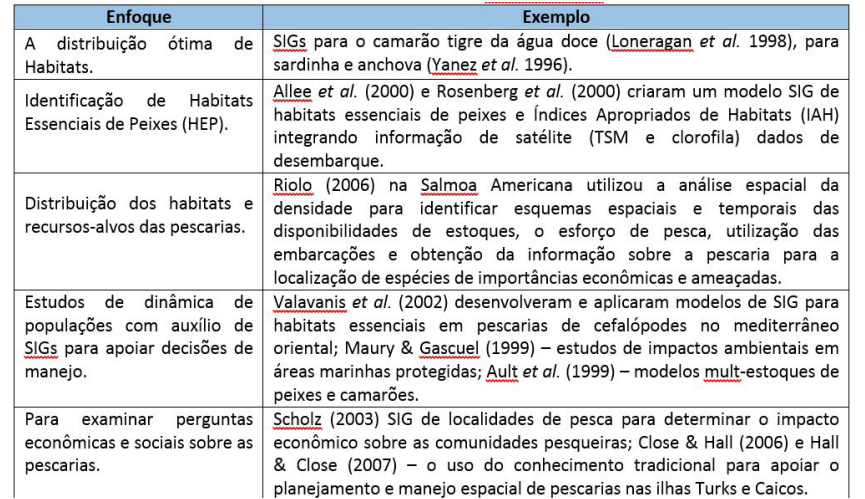

Tabela 1: Síntese de algumas das linhas de estudo da geoinformação com enfoque pesqueiro.

Fonte: (SOUSA, 2009).

Esses exemplos servem para enfatizar a importância de se promover a atuação desses conhecimentos nos recursos pesqueiros, através dos Sistemas Integrados de Geoinformação Pesqueira (SIGPe) (SOUSA, 2009).

"A utilização desses sistemas torna possível criar cenários para despertar o pensamento crítico, em que o aluno deverá buscar suas próprias respostas, e não recebêlas prontas, ditas como verdades absolutas" (SONSOLO e BACK, 2008, p.5).

Os SIG constituem tecnologia e método para analisar diversas variáveis ambientais de caráter espacial. Através da análise dos fenômenos, eles auxiliam na compreensão de padrões, relações e tendências que ocorrem na superfície do planeta em diversas escalas espaciais, contribuindo para os profissionais que atuam, diretamente, com recursos naturais. "A sua utilização como ferramenta de ensino em diferentes localidades tem apresentado, entre outros, uma melhoria na atitude dos estudantes frente à tecnologia e na análise de dados geográficos” (BAKER e WHITE apud SILVA 2005, p. 1) “[...] e um maior engajamento dos estudantes no desenvolvimento de habilidades mentais avançadas enquanto investigam

A perspectiva geográfica passa por uma alta demanda, em parte por causa do sucesso dos usuários de SIG na solução de problemas. 0 mesmo autor recomenda que ensinar com o auxílio dos SIGs seja utilizado como método de integração do pensamento geográfico em outras disciplinas além da Geografia (Kerski apud SILVA 2005, p. 2).

Assim, podemos dizer que a utilização da geotecnologia como mediação pedagógica no mencionado curso técnico tende a ampliar as atividades que despertam o interesse em aprender, possibilitando informações georreferenciadas de grande valia, como evidencia Silva (2005, p. 2):

O poder de um SIG [...] está relacionado ao fato de que o mesmo permite e nos leva ao questionamento dos dados. Logo, a sua implementação dentro das atividades de ensino pode encorajar os estudantes a examinar dados de vários campos da ciência. Dentro dessa abordagem interrogativa, os estudantes, então, desenvolvem questões de pesquisa, metodologias, obtêm e analisam dados, gerando suas próprias conclusões.

Desse modo, seria significativo para o desenvolvimento cognitivo dos futuros profissionais da área o uso dessas ferramentas em sua formação, até porque "O SIG auxilia no planejamento e na escolha de locais adequados para o cultivo de organismos marinhos (maricultura). A relação de dados espaciais com a cartografia temática nesse caso permite velocidade e precisão para a tomada de decisões" (GUNEROGLU et al. apud BOSCATTO 2007, p. 1680). 
"O SIG vem sendo utilizado nos últimos anos, com frequência nos estudos terrestres. Porém pesquisas direcionadas a disciplina no ambiente marinho vem apresentando resultados em diversas regiões do planeta" (LEHAMANN e LACHAVANNE, et. al. apud BOSCATTO, 2007, p. 1680). "A cartografia e o SIG puderam demostrar que são capazes de agilizar o processamento de dados e a representação em forma de mapas para distintos trabalhos na zona marinha, tanto no zoneamento quanto na escolha de locais para produção de organismos aquáticos" (BOSCATTO, 2007, p. 1680).

Como visto, há uma grande investida no estudo de geoprocessamento nas mais diversas áreas de conhecimento, gerando um leque de possibilidades e de diferencial para o profissional que faz uso dessa ferramenta. Vários estudos relatam também sua importância no eixo de recursos naturais, em especial, no que foi possível observar quanto ao crescente investimento desse mercado aplicado às práticas de recursos pesqueiros. Assim, dada à relevância de se trabalhar essa temática no IFRN/Campus Macau, foi aprovado concomitantemente a nossa pesquisa, o projeto de extensão "O Manguezal de Macau/RN visto do espaço: uma nova abordagem na disseminação do conhecimento geotecnológico ambiental para estudantes do curso Técnico em Recursos Pesqueiros". Esse projeto de extensão foi uma abordagem de ensino, financiada pelo edital N 01/2014 da Pró-reitoria de Extensão (PROEX) do IFRN.

0 respectivo projeto, de viés inovador, permitiu a obtenção de dados, junto a alguns docentes do referido curso, além da utilização de uma turma, como modelo, para a inserção do estudo de geotecnologias. Seu conjunto de atividades possibilitou, a essa turma e à comissão do curso, uma experiência única, atestando o interesse dos alunos para o uso dessas novas ferramentas geotecnológicas. Além disso, essa experiência propiciou aos futuros técnicos ganharem, em sua formação, um conjunto de habilidades adicionais que Ihes permite desempenhar uma série de atividades com mais exatidão na obtenção de dados, análises etc. Os profissionais formados nessa perspectiva terão, com isso, condições mais expressivas de executar suas funções com mais qualidade e controle de recursos, destacando-se no mundo profissional.

\section{O INSTITUTO FEDERAL DE EDUCAÇÃO, CIÊNCIA E TECNOLOGIA DO RIO GRANDE DO NORTE - IFRN/CAMPUS MACAU}

O IFRN é uma instituição de ensino reconhecida em todo o território nacional que, há mais de um século, oferta educação de qualidade na formação de profissionais nas mais diversas áreas do mercado de trabalho. Sua função é ofertar

[...] educação profissional e tecnológica - de qualidade referenciada socialmente e de arquitetura político-pedagógica capaz de articular ciência, cultura, trabalho e tecnologia - comprometida com a formação humana integral, com o exercício da cidadania e com a produção e a socialização do conhecimento, visando, sobretudo, a transformação da realidade na perspectiva da igualdade e da justiça sociais (IFRN, 2012a, p. 21).

O IFRN/Campus Macau, onde o presente trabalho foi desenvolvido, constitui uma instituição que integra a segunda fase do Plano de Expansão da Rede Federal de Educação Profissional e Tecnológica do Ministério da Educação, iniciada no ano de 2007. Estruturalmente esse Campus possui uma área de aproximadamente $290 \mathrm{~km}^{2}$ e está situado na cidade-polo da microrregião salineira, Macau-RN, distando 176 km da capital do 
estado. Este município é um dos maiores produtores de sal do Brasil e o primeiro produtor de petróleo do Estado em exploração marítima, além de ser o segundo maior produtor de pescado do Rio Grande do Norte.

Esse campus conta, atualmente, com os cursos: Técnicos em Recursos Pesqueiros; em Química; e em Informática; Licenciatura em Biologia e Especialização em Ensino de Ciências Naturais e Matemática. Além disso, desenvolve diferentes projetos, como, por exemplo, o Encontro de Pescadores da Microrregião Salineira, que conta com exposições e oficinas como a de "Segurança de pescadores", ministrada por representantes da Marinha do Brasil.

\section{O CURSO TÉCNICO EM RECURSOS PESQUEIROS DO IFRN/CAMPUS MACAU}

O Curso Técnico em Recursos Pesqueiros (RP) no IFRN é ofertado apenas no Campus Macau, como Ensino Médio Integrado, quando os conhecimentos técnicos da área apresentam-se interligados aos conteúdos básicos desta etapa de ensino; e na forma Subsequente, destinado aos alunos que já concluíram o ensino médio, sendo ambos ministrados na modalidade presencial. Esse curso apresenta como objetivo geral formar técnicos de nível médio com

[...] competência técnica, ética e política para desempenhar suas atividades profissionais, nas atividades de captura e de cultivo de organismos que tenham como principal habitat a água para seu aproveitamento integral na cadeia produtiva, com segurança de qualidade e sustentabilidade econômica, ambiental e social (IFRN, 2012b, p. 7).

Sua organização curricular observa as determinações presentes na Lei nº 9.394/96, alterada pela Lei $n^{\circ} 11.741 / 2008$, nas Diretrizes Curriculares Nacionais da Educação Profissional Técnica de Nível Médio, bem como nos princípios e diretrizes definidos no Projeto Político-Pedagógico - PPP - do IFRN. E sua matriz curricular, no modo Subsequente, está organizada por disciplinas em regime seriado semestral, com uma carga-horária total 1.760 horas, sendo 1.260 horas destinadas às disciplinas, 100 horas aos seminários curriculares e 400 horas à prática profissional. A tabela 2 descreve a composição dessa matriz curricular.

Nessa composição, as disciplinas encontram-se articuladas, fundamentadas nos conceitos de interdisciplinaridade e contextualização. Centram-se nos perfis profissionais de conclusão estabelecidos no Projeto Pedagógico do Curso que ensejam a formação integrada articulando ciência, trabalho, cultura e tecnologia, bem como a aplicação de conhecimentos teórico-práticos específicos do eixo tecnológico e da habilitação específica. 


\begin{tabular}{|c|c|c|c|c|c|c|}
\hline \multirow[t]{2}{*}{ DISCIPLINAS } & \multicolumn{4}{|c|}{$\begin{array}{l}\text { Número de aulas semanal } \\
\text { por Série/Semestre }\end{array}$} & \multicolumn{2}{|c|}{$\begin{array}{c}\text { Carga-horária } \\
\text { total }\end{array}$} \\
\hline & $1^{\circ}$ & $2^{\circ}$ & $3^{\circ}$ & $4 \stackrel{9}{2}$ & Hora / aula & Hora \\
\hline \multicolumn{7}{|l|}{ Núcleo Fundamental } \\
\hline Língua Portuguesa & 4 & & & & 80 & 60 \\
\hline Inglês & 2 & & & & 40 & 30 \\
\hline Espanhol & 2 & & & & 40 & 30 \\
\hline Matemática & 4 & 0 & 0 & 0 & 80 & 60 \\
\hline Subtotal de carga-horária do núcleo fundamental & 12 & 0 & 0 & 0 & 240 & 180 \\
\hline \multicolumn{7}{|l|}{ Núcleo Articulador } \\
\hline Informática* & 3 & & & & 60 & 45 \\
\hline Filosofia, Ciência e Tecnologia & & 2 & & & 40 & 30 \\
\hline Sociologia do Trabalho & & & 2 & & 40 & 30 \\
\hline Qualidade de Vida e Trabalho & & & & 2 & 40 & 30 \\
\hline Gestão Organizacional e Empreendedorismo* & & & & 3 & 60 & 45 \\
\hline Física Aplicada & 2 & & & & 40 & 30 \\
\hline Relações Interpessoais do trabalho & & 2 & & & 40 & 30 \\
\hline Subtotal de carga-horária do núcleo articulador & 5 & 4 & 2 & 5 & 320 & 240 \\
\hline \multicolumn{7}{|l|}{ Núcleo Tecnológico } \\
\hline Associativismo e Cooperativismo & & & 2 & & 40 & 30 \\
\hline Biologia Pesqueira & & 2 & & & 40 & 30 \\
\hline Carcinicultura, Algocultura e Ranicultura & & & & 4 & 80 & 60 \\
\hline Equipamentos Eletrônicos de Apoio e Navegação & & 4 & & & 80 & 60 \\
\hline Extensão Pesqueira & & & 2 & & 40 & 30 \\
\hline Instalações Elétricas e Hidráulicas em Embarcações & & 3 & & & 60 & 45 \\
\hline Introdução a Pesca e Aquicultura & 2 & & & & 40 & 30 \\
\hline Máquinas e Motores marítimos & & & 3 & & 60 & 45 \\
\hline Marinharia e Confecção de Apetrechos de Pesca & 2 & & & & 40 & 30 \\
\hline Meio Ambiente, Desenvolvimento e Sustentabilidade & & & & 2 & 40 & 30 \\
\hline Meteorologia e Climatologia* & & 4 & & & 80 & 60 \\
\hline Oceanografia e Limnologia & & & 4 & & 80 & 60 \\
\hline Operações com Embarcações & & & & 2 & 40 & 30 \\
\hline Piscicultura e Malococultura & & & 4 & & 80 & 60 \\
\hline Segurança no Trab., Primeiros Socorros e Salvatagem & & & & 4 & 80 & 60 \\
\hline Tecnologia do Pescado & & & & 4 & 80 & 60 \\
\hline Tecnologia Pesqueira* & & & 4 & & 80 & 60 \\
\hline Topografia e Construções Aquícolas & & 4 & & & 80 & 60 \\
\hline Subtotal de carga-horária do núcleo tecnológico & 4 & 17 & 19 & 16 & 1.120 & 840 \\
\hline Total de carga-horária das disciplinas & 21 & 21 & 21 & 21 & 1.680 & 1.260 \\
\hline
\end{tabular}

Tabela 2: Matriz Curricular do Curso de Técnico Subsequente em Recursos Pesqueiros. Fonte: (IFRN, 2012b)

Segundo nossa concepção, um componente que abordasse o estudo das geotecnologias aplicadas aos RP comporia essa matriz curricular no grupo das disciplinas do núcleo tecnológico. Ou, mediante a impossibilidade de sua inclusão como componente curricular, a temática poderia ser inserida em algum(ns) dos demais componentes já existentes, tais como: Topografia e Construções Aquícolas; Equipamentos Eletrônicos de Apoio e Navegação; Tecnologia Pesqueira; entre outros. 
O técnico em Recursos Pesqueiros, enquanto um profissional que recebe uma formação do eixo tecnológico "Recursos Naturais", deve voltar-se ao uso desses recursos em prol do melhor desenvolvimento do seu trabalho e da sustentabilidade local e global. Para isso precisa fazer um levantamento dos recursos naturais existentes nas suas áreas de atuação e a forma como esses recursos estão sendo utilizados, de modo a promover o bom rendimento e o cuidado com o meio natural. Neste caso, o uso de tecnologias pode servir como um subsídio essencial, e dentre eles, os conhecimentos geotecnológicos, pois estes podem contribuir para o processo de planejamento, a aquisição, a análise e o processamento de dados acerca desse meio para a atuação profissional. Dada sua importância, pretende-se aqui, avaliar a viabilidade do ensino de geoprocessamento no Curso Técnico em Recursos Pesqueiros do IFRN/Campus Macau.

\section{CONCEPÇÃO DE CURRÍCULO: CURSO TÉCNICO EM RECURSOS PESQUEIROS}

Em vista das considerações até então apresentadas, evidenciamos como proposição deste trabalho visitar trechos da organização curricular do IFRN visando destacar os itens que servem de embasamento a nossa proposta em relação ao curso enfatizado. A este respeito é importante evidenciar que o IFRN traz como definição de currículo em seu PPP (IFRN, 2012a), a ideia de

Um conjunto integrado e articulado de atividades intencionadas, pedagogicamente concebidas a partir da visão crítica de ser humano, de mundo, de sociedade, de trabalho, de cultura e de educação, organizadas para promover a construção, a reconstrução, a socialização e a difusão do conhecimento (IFRN, 2012a, p. 49).

No que diz respeito à Educação Profissional, o documento pauta-se

[...] pelo entendimento de que a formação geral é parte inseparável da formação para o trabalho em todos os processos educativos e produtivos. A esse modelo está vinculado [...] o direito a uma formação ampla que possibilite leitura crítica do mundo, atuação como cidadão e emancipação humana. (CIAVATTA apud IFRN, 2012a, p. 52)

Nessa perspectiva, a educação profissional ofertada pela instituição volta-se à formação para uma leitura crítica do mundo, visando uma constituição cidadã e, conjuntamente, um conhecimento que lhe propicie domínio no âmbito profissional.

Sobre a constituição dos cursos técnicos de nível médio, a instituição assim se posiciona:

[...] trata-se de uma concepção curricular que favorece o desenvolvimento de práticas pedagógicas integradoras e articula o conceito de trabalho, ciência, tecnologia e cultura, à medida que os eixos tecnológicos se constituem de agrupamentos dos fundamentos científicos comuns, de intervenções na natureza, de processos produtivos e culturais, além de aplicações científicas às atividades humanas. (IFRN, 2012b, p. 9) 
A proposta desses cursos detém-se, portanto, no estímulo à interdisciplinaridade, reconhecendo a necessidade de uma educação profissional que integre conhecimentos e demais experiências advindas do mundo profissional; além de impulsionar uma relação que possibilite a construção do pensamento tecnológico crítico e a capacidade de intervir em situações concretas.

Essa configuração destaca, assim, abordagens diversas surgidas nos últimos anos em defesa de revisões curriculares que pudessem acompanhar as evoluções propostas pelo mundo do trabalho, mas também vinculadas à ideia da formação integral, algo essencial para uma formação profissional de qualidade.

\section{MATERIAL E MÉTODOS}

Inicialmente o presente trabalho desenvolveu análises de estudos de casos através de um levantamento bibliográfico. Segundo Gil (apud AQUINO 2014, p. 26), a pesquisa bibliográfica

[...] é desenvolvida a partir de material já elaborado, constituído principalmente de livros e artigos científicos [...] A principal vantagem de uma pesquisa bibliográfica reside no fato de permitir ao investigador a cobertura de uma gama de fenômenos mais ampla do que aquela que poderia pesquisar diretamente (GIL apud AQUINO, 2014, p. 26).

Nesse momento foram analisados estudos do campo das geotecnologias. Vimos resultados de Babcock et. al. (2005), Baker e White (2003), Boscatto (2007), Fisher e Toepfer (1998), Fisher e Rahel (2004), Guneroglu et. al. (2005), Kerski (2003), Morris e Ball (2006), Silva (2005), Sonsolo e Back (2008), Sousa (2009) e West (2003) e fizemos uso de seus dados visando destacar a existência de trabalhos e demais linhas de pesquisas, em escala mundial, que usam geotecnologias atreladas as suas áreas de atuação, além das vantagens proporcionadas por isso.

Feito isso, elaboramos uma pesquisa empírica "uma vez que foi necessária à coleta de dados em campo" (AQUINO, 2014, p. 23). Para tanto apresentamos nossa proposta ao corpo docente do referido curso, visando perceber a concepção dos docentes sobre a temática, além de analisar o conhecimento dos mesmos acerca do assunto. Tal realidade configurou, assim, uma abordagem qualitativa da pesquisa. Nesse tipo de abordagem é preciso que o investigador "vá a campo, previamente munido de um referencial teórico e suas devidas inquietações acerca do objeto de pesquisa, deixando que os acontecimentos ocorridos durante a pesquisa leve-o aos resultados, positivos ou negativos" (AQUINO, 2014, p. 25).

Na sequência das realizações, o início do segundo semestre de 2014 foi marcado pela aplicação de um questionário a um grupo amostral de 20 (vinte) docentes, abordando profissionais das áreas de: Administração, Aquicultura, Bacias Hidrográficas, Biologia Evolutiva, Biologia Marinha, Comunidades Étnicas, Direito Ambiental, Educação, Engenharia de Pesca, Geografia, História, Matemática, Meio Ambiente, Operação em Embarcações, Química, Recursos Pesqueiros, Segurança do Trabalho e Sociologia, ativos no IFRN/Campus Macau.

A respeito dessa técnica para o levantamento de dados, Severino (apud AQUINO 2014, p. 28) a descreve como "[...] um conjunto de questões, sistematicamente articuladas, que se destinam a levantar informações escritas por parte dos sujeitos pesquisadores, com vistas a conhecer a opinião dos mesmos, sobre os assuntos em estudo." 
O questionário aplicado buscou levantar o conhecimento dos docentes participantes sobre a temática de geoprocessamento e de sua importância no trabalho dos futuros técnicos em recursos pesqueiros; as opiniões dos mesmos sobre a inserção dessa temática aos componentes curriculares do referido curso, bem como sobre a forma como tal saber poderia ser desenvolvido.

\section{RESULTADOS E DISCUSSÃO}

Os 20 docentes participantes da pesquisa representam 31,74\% da equipe de professores do respectivo Campus. Todos eles ministram disciplinas no Curso Técnico em Recursos Pesqueiros e constituem 18 áreas de atuação, conforme a Figura 1.

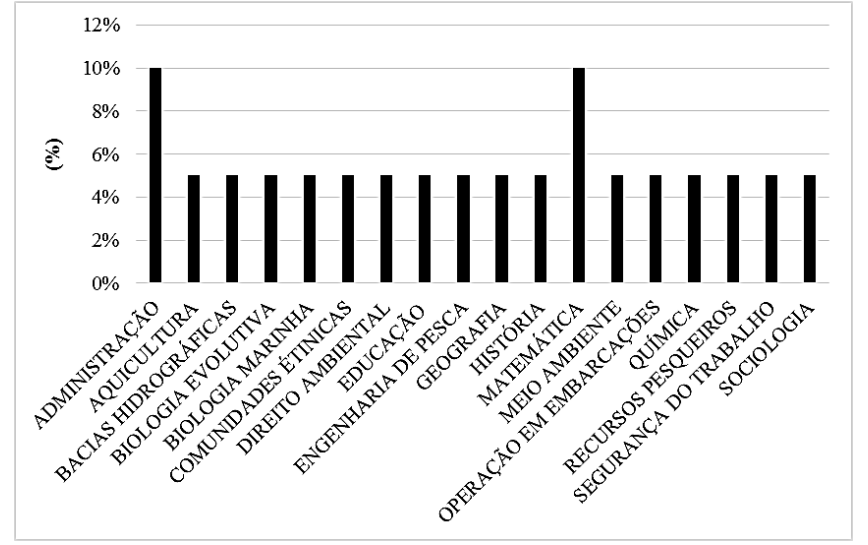

Figura 1: Representativo da área de atuação dos professores participantes.

A pesquisa revela, assim, uma abrangência multidisciplinar, visando não favorecer respostas por uma área específica. Além disso, 70\% dos participantes estão entre a titulação de Mestre e Doutor (Figura 2). Esse resultado demonstra que os envolvidos possuem um relevante conhecimento específico de suas respectivas áreas.

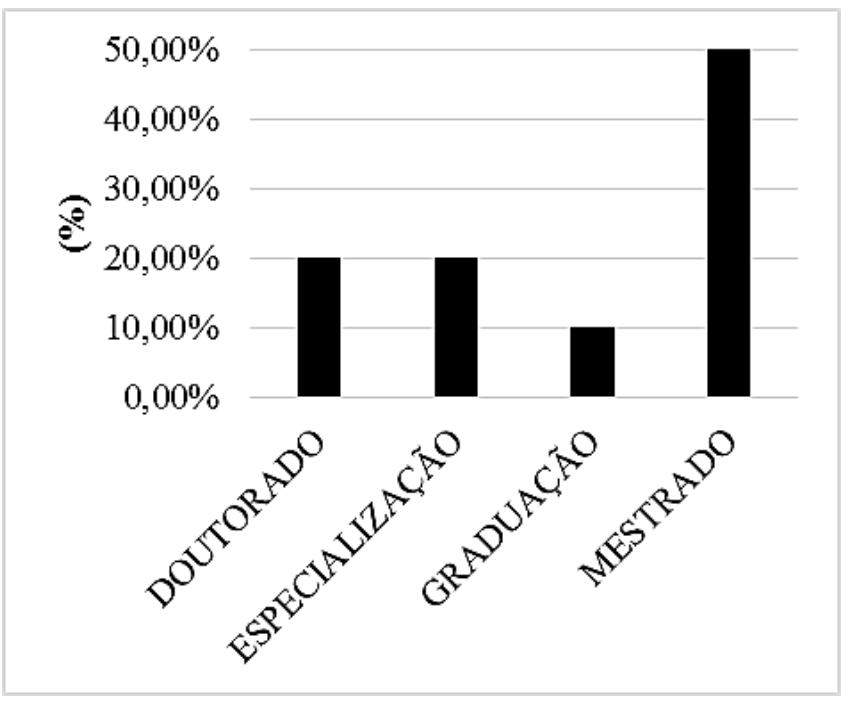

Figura 2: Representativo da titulação dos professores participantes

Ao serem questionados sobre o conhecimento que possuíam em relação ao geoprocessamento, $50 \%$ dos participantes relataram possuir um conhecimento que, em escala, distribui-se entre moderado a avançado, enquanto os demais afirmaram desconhecer a temática (Figura 3). Considerando que na amostragem utilizada constaram profissionais de áreas diversas, alguns deles que não trabalham especificamente com esse tipo de tecnologia, torna-se explicável a falta de entendimento sobre a temática por parte desses profissionais. 


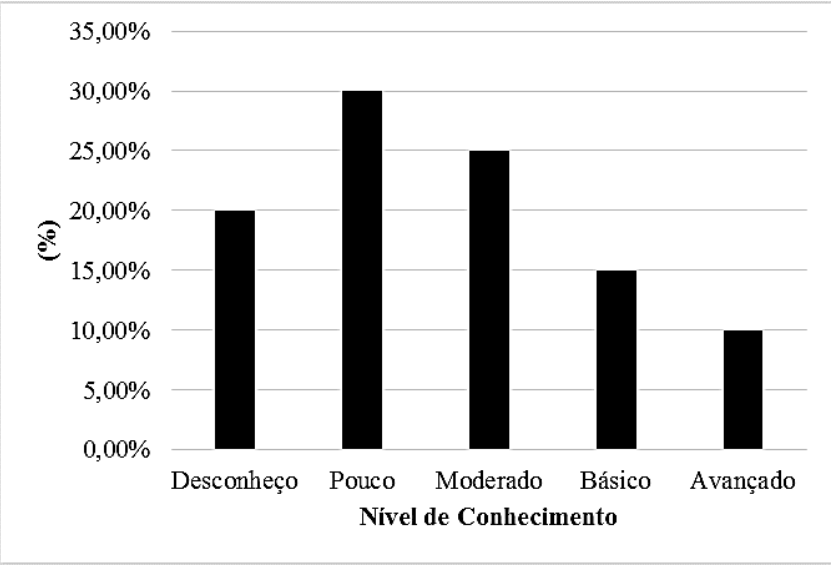

Figura 3: Representativo do conhecimento dos participantes sobre o geoprocessamento.

Acerca da utilização de técnicas de geoprocessamento no estudo de recursos pesqueiros (Figura 4), 70\% dos professores confirmaram já terem tido acesso a esse tipo de informação.

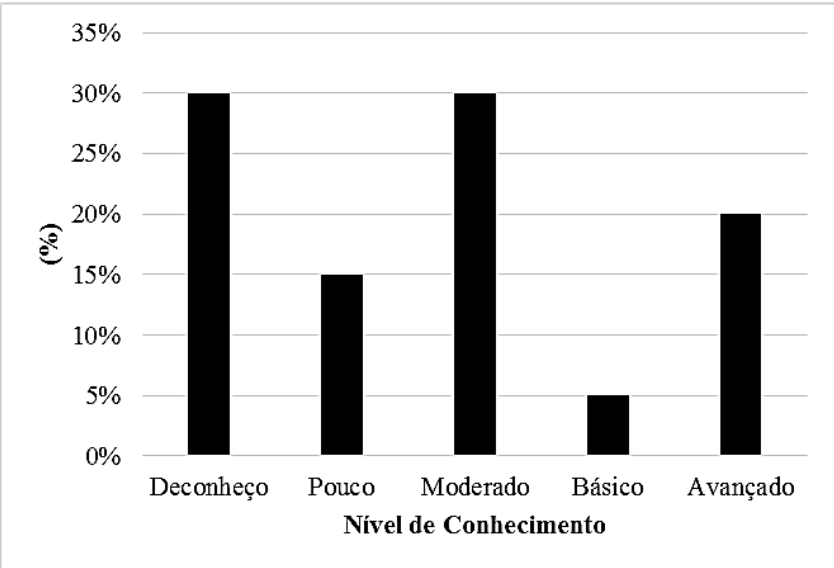

Figura 4: Representativo do conhecimento dos participantes sobre o geoprocessamento aplicado ao estudo de Recursos Pesqueiros.

Em relação à participação em algum curso referente ao uso de ferramentas geotecnológicas, $65 \%$ dos participantes responderam positivamente. Essa afırmativa reforça a importância que esse tipo de tecnologia tem em nosso cotidiano, posto que mesmo diante da variedade de áreas a que se ligam os participantes, o geoprocessamento vem sendo contemplado por mais da metade delas, o que lhe dá ênfase em meio às grandes tecnologias atuais, possuindo um vasto campo de conhecimento, conforme destacam Sonsolo e Back (2008).

Sobre a importância dessa abordagem, mais de 55\% dos participantes acreditam que esse tipo de estudo é de grande relevância nas mais diversas áreas de estudo (Figura 5).

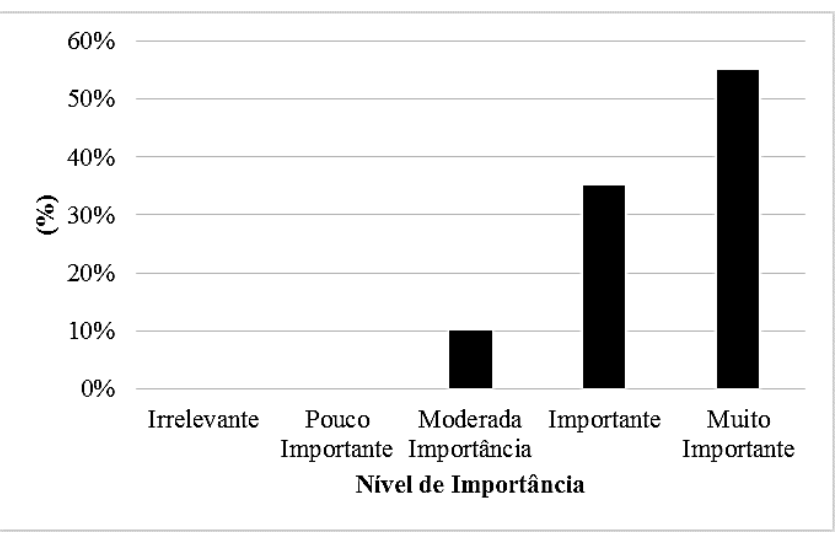

Figura 5: Indicativo da opinião dos participantes sobre a importância de se trabalhar o geoprocessamento no Curso Técnico em Recursos Pesqueiros. 
A temática assume uma relevância ainda maior por trabalhar com a construção de modelos espaciais que contribuem com estudos em diversas áreas. De acordo com Alves (apud SONSOLO e BACK, 2008, p. 5-6): "modelos nos permitem simular o que deverá acontecer, sob certas condições. Com o seu auxílio simulamos situações, sem que elas jamais aconteçam. Isto nos permite ajustar o comportamento ou para evitar, ou para provocar um determinado futuro."

Se começássemos a instigar nossos alunos à construção de maquetes cada vez mais bem elaboradas, e se com o auxílio da geotecnologia essas maquetes pudessem evoluir para modelos virtuais com um dimensionamento espacial cada vez mais próximo da realidade, essa constante atração pelo "novo" possibilitaria resultados bem significativos a nossa proposta.

Ao serem questionados sobre a recepção dos alunos acerca da inserção desse novo conhecimento ao Curso Técnico em RP, 85\% dos participantes afirmaram que isto poderia percorrer uma escala que iria de moderado a muito bem recebido (Figura 6). Quanto a isso, devemos considerar que embora uma parcela dos alunos não goste de trabalhar com recursos que utilizem um determinado conhecimento matemático ou geográfico, eles são, no entanto, atraídos pelas tecnologias e suas evoluções.

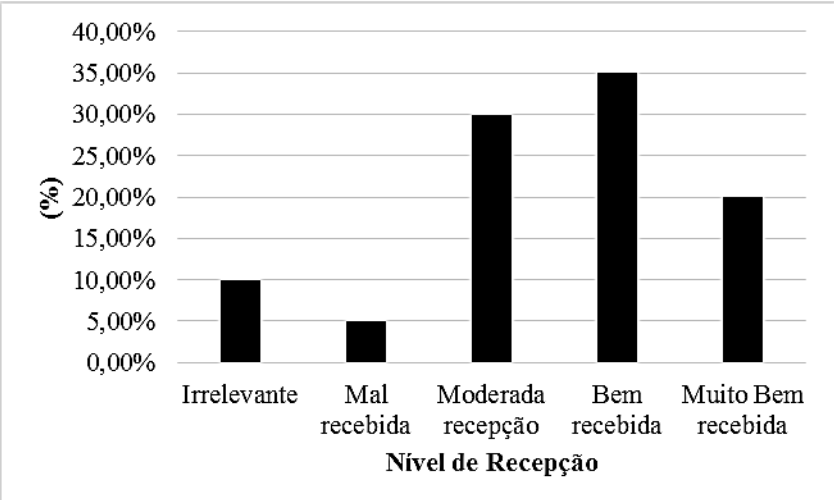

No que tange ao nível de contribuição que esse novo conhecimento poderia possibilitar aos futuros técnicos em RP, 70\% dos participantes da pesquisa acreditam que seria excelente essa contribuição, causando um diferencial na formação desses profissionais (Figura 7).
Figura 6: Indicativo da opinião dos participantes sobre a recepção dos alunos quanto à inserção de geoprocessamento no curso Técnico em Recursos Pesqueiros.

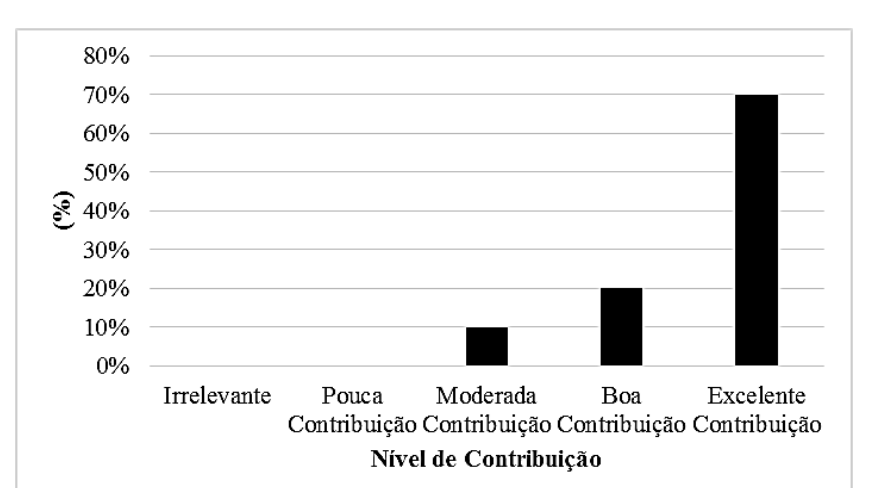

Figura 7: Indicativo da opinião dos professores participantes sobre a contribuição do geoprocessamento para a formação dos futuros técnicos em Recursos Pesqueiros.

Conforme discutido ao longo desta produção, 
A utilização dos SIGs como ferramenta de ensino em diferentes regiões no mundo, tem apresentado uma melhoria na desenvoltura dos estudantes frente à tecnologia e na análise de dados geográficos, proporcionando um maior engajamento dos mesmos no desenvolvimento de habilidades mentais avançadas enquanto investigam assuntos de interesse pessoal. (BAKER e WHITE 2003; WEST apud SILVA 2005, p. 1)

Ainda nos posicionamentos dos entrevistados, $100 \%$ deles acreditam que esse tipo de abordagem possibilitará aos futuros profissionais um diferencial significativo em suas futuras práticas no mundo do trabalho e $90 \%$ dos mesmos defendem a necessidade de receberem uma formação específica mediante a inserção desse tipo de conhecimento no referido curso. Segundo a análise, $100 \%$ dos participantes afirmam a necessidade de uma revisão curricular do curso de modo a contemplar a inserção do conhecimento geotecnológico.

Diante da importância dos dados revelados, questionamos, por fim, sobre a melhor forma de promover a adição do geoprocessamento no curso de RP (Figura 8). Sobre isto, $40 \%$ acreditam que o melhor método seria inseri-lo como um novo componente curricular; $25 \%$ explicam que esse tipo de conhecimento pode ser trabalhado em forma de curso de extensão; outros $25 \%$ apostam em sua oferta como uma disciplina optativa; e os $10 \%$ restantes percebe que a temática pode encontrar-se disseminada em meio ao estudo das disciplinas. Observamos que apesar da diversidade, mostra-se importante considerar a relevância das opiniões apresentadas.

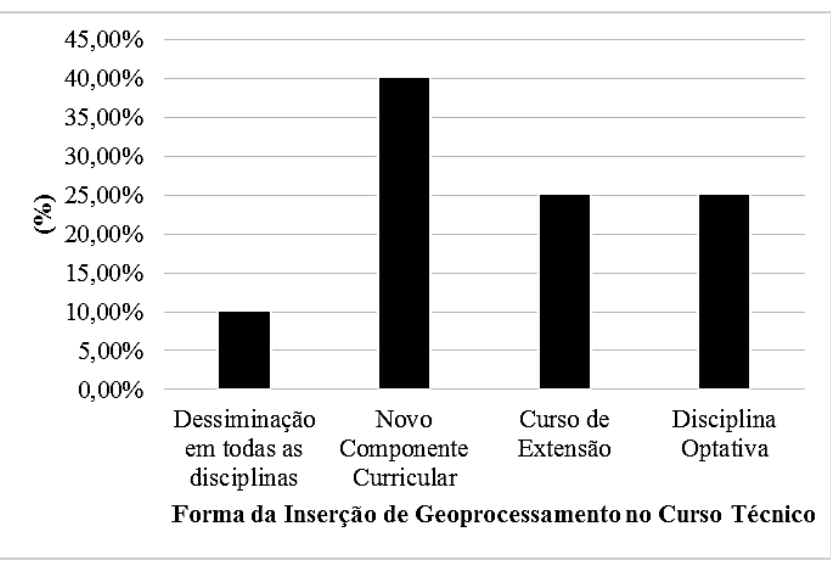

Figura 8: Indicativo da opinião dos participantes sobre a forma mais adequada deinserir o conhecimento do geotecnológico ao Curso Técnico em Recursos Pesqueiros.

\section{CONCLUSÃO}

No decorrer deste estudo, foram encontrados diversos trabalhos científicos que evidenciaram a importância da abordagem geotecnológica no curso técnico em Recursos Pesqueiros. Foi comprovada a disseminação desse tipo de ferramenta em uma grande variedade de áreas de estudo, principalmente a sua utilização no que converge à área de RP.

Relatos de alguns autores sobre a contribuição que o estudo do SIG tende a proporcionar para os estudantes em sua rotina de formação também foram constatados, enriquecendo ainda mais a defesa dessa proposta, sobretudo, pela forma como essa tecnologia se expandiu pelo mundo e chegou ao nosso país, propiciando novas possibilidades em várias áreas. 
Através de trabalhos pesquisados, comprovamos a necessidade de revisões nas matrizes curriculares das instituições de ensino, em especial dos cursos técnicos, de modo que essas matrizes acompanhem as evoluções que acometem o mundo do trabalho. Embora não seja uma tarefa fácil acompanhar esse avanço, faz-se necessária sempre uma iniciativa, por parte dessas instituições, nessa direção.

Ao analisar o currículo do curso de RP do IFRN campus Macau (IFRN, 2012b) e do próprio PPP do IFRN (IFRN, 2012a), estabelecemos uma relação direta entre essa iniciativa que vem se expandindo e a sugestão de revisão curricular por nós defendida, tendo em vista que propomos aqui a inserção do estudo de uma ferramenta tecnológica que está em alta em escala mundial, tendo como justificativa a ideia de que a mesma proporciona melhores condições de empregabilidade aos futuros técnicos formados pela instituição, apresentando-os como um diferencial em meio ao dinâmico e cada vez mais exigente mercado de trabalho.

A aplicação do questionário com os professores do curso de Recursos Pesqueiros do IFRN/Campus Macau foi de grande valia em nossa pesquisa. Através da análise dos dados podemos comprovar o quanto é justificável a inserção desse conhecimento no referido curso. A opinião dos mesmos, em todos os pontos analisados, confirmou a necessidade de uma adequação na matriz curricular do curso visando contemplar o estudo geotecnológico, seja como um novo componente curricular ou mesmo um conteúdo nas disciplinas componentes do curso.

A realização das oficinas mostrou-se de grande relevância também, uma vez que "simulou" a receptividade dos discentes em meio à inserção dessa nova proposta de ensino na forma de um componente curricular. Durante a realização das mesmas, os discentes tiveram a oportunidade de conhecer, basicamente, sobre essa ferramenta, assim como suas funcionalidades enquanto um diferencial para suas vidas profissional e acadêmica. Ao término de cada uma das oficinas, foi notável o interesse dos participantes em adquirir um maior conhecimento acerca das múltiplas possibilidades das geotecnologias.

Esse estudo deverá contribuir como embasamento para futuras tomadas de decisão, na gestão do Curso Técnico em Recursos Pesqueiros do IFRN, de modo a identificar a real viabilidade desse processo e a forma mais efetiva de sua aplicação. Além disso, poderá ser norteador para o incremento do conhecimento geotecnológico nos cursos Técnicos em Recursos Pesqueiros dos Institutos Federais no Brasil.

\section{REFERÊNCIAS BIBLIOGRÁFICAS}

1. ALLEE, R. J., DETHIER, M., BROWN, D., DEEGAN, L., FORD, R. G., HOURIGAN, T. F., MARAGOS, J., SCHOCH, C., SEALEY, K., TWILLEY, R., WEINSTEIN, M. P. \& YOKLAVICH, M. Marine and estuarine ecosystem and habitat classification. NOAA Technical Memorandum, NMFSeF/SPOe43. US NOAA National Marine Fisheries Service. 2000.

2. AQUINO, Javaerton de Souza. O FUNDEB e o Conselho de Controle Social: A Participação dos Conselheiros do Fundo no Município de Macau/RN. 2014. 136 f. Monografia (Graduação) - Curso de Licenciatura em Biologia, Instituto Federal de Educação, Ciências e Tecnologia do Rio Grande do Norte, Macau, 2014.

3. AULT, J. S., LUO, J., SMITH, S. G., SERAFY, J. E., WANG, J.D., HUMSTON, R. \& DIAZ, G. A. A spatial dynamic multistock production model. Canadian Journal of Fisheries and Aquatic Sciences, 56: 4-25. 1999. 
4. BABCOCK, E. A., PIKITCH, E. K., MC ALLISTER, M. K., APOSTALAKI, P. \& SANTORA, C. A. Perspective on the use of spatialized indicators for ecosystem-based fishery management through spatial zoning. ICES Journal of Marine Science, 62: 469-76. 2005.

5. BAKER; WHITE. The effects of GIS on students attitudes, selfefficacy, and achievement in middle school science classrooms. Journal of Geography. V.102, n.6, p. 243254. 2003.

6. BOSCATTO; PROCHNOW; OLIVEIRA, 21 a 24 de Outubro de 2007. A Utilização do Sistema de Informações Geográfica - SIG como apoio ao planejamento legal da Malacocultura. XXIII Congresso Brasileiro de Cartografia, Rio de Janeiro, Brasil.Close \& Hall (2006)

7. FISHER; RAHEL. Geography Information Systems in Fisheries. American Fisheries Society, Bethesda, Maryland, 275p. 2004.

8. FISHER; TOEPFER. Recent trends in geographic information systems education and fisheries research applications at U.S. Universities. Fisheries, 23:10-13. 1998.

9. GUNEROGLU, A; E. KOSE, C. ERUZ, E. BASAR, S. ERKEBAY E F. KARSLI. Use of geografic information system (GIS) to select fish cage farming sites in Surmene Bay, Black Sea. The Israeli Journal of Aquaculture, Vol. 57. N², pp.81-89. 2005.

10. HALL, G. B. \& CLOSE, C. H. Local knowledge assessment for a small-scale fishery using geographic information systems. Fisheries Research, 83: 11-22. 2007.

11. IFRN. Projeto Político-Pedagógico do IFRN: uma construção coletiva. Natal, 2012. Disponível em: <http://www.ifrn.edu.br>. Acesso em: 18 jun. 2015.

12. IFRN. Projeto Pedagógico do Curso Técnico de Nível Médio em Recursos Pesquiros na forma Subsequente, na modalidade presencial. Natal, 2012. Disponível em: <http://www.ifrn.edu.br>. Acesso em: 18 jun. 2015.

13. KERSKI, J. The implantation and effectiveness of Geographic Information Systems technology in secondary education. Journal of Geography, v. 102, n.3, p. 128-137. 2003.

14. LONERAGAN N.R., KENYON R.A., STAPLES D.J., POINER I.R. AND CONACHER C.A. The influence of seagrass type on the distribution and abundance of postlarval and juvenile tiger prawns (Penaeus esculentus and P. semisulcatus) in the western Gulf of Carpentaria, Australia. Journal of Experimental Marine Biology and Ecology 228, 175-195. 1998.

15. MAURY, O. \& GASCUEL, D. S. Simulateur halieutique de dynamiques spatiales, a GIS based numerical model of fisheries. Example application: the study of a marine protected area. Aquatic Living Resources, 12: 77-88. 1999.

16. MORRIS, L. \& BALL, D. Habitat suitability modelling of economically important fish species with commercial fisheries data. ICES Journal of Marine Science, 63: 1590-1603. 2006.

17. RIOLO, F. A geographic information system for fisheries management in American Samoa. Environmental Modeling Software, 21: 1025-1041. 2006.

18. ROSENBERG, M.S., ADAMS, D.C., GUREVITCH, J. MetaWin: Statistical Software for Meta-Analysis, Version 2. Sinauer Associates, Sunderland, MA, USA. 2000. 
19. SCHOLZ, A. J. Groundfish Fleet Restructuring Information and Analysis Project: Final Report and Technical Documentation. Pacific Marine Conservation Council/Ecotrust, San Francisco, California, 63p. 2003.

20. SILVA, Tatiana S. O Uso dos Sistemas de Informação Geográfica para o Ensino em Recursos Pesqueiros. CINTED-UFRGS Revista Novas Tecnologias na Educação. v. 3. Nº 1, Maio, 2005.

21. SONSOLO, Davis Gruber; BACK, Gilberto. O Ensino de Geoprocessamento Para Estudantes de Turismo: Uma Discussão Sobre suas Potencialidades. V Seminário de Pesquisa em Turismo do MERCOSUL (SeminTUR), 27 e 28 de Junho de 2008, pp. 1-7.

22. SOUSA, Keid Nolan Silva. Representação espacial de dados pesqueiros na Costa Norte Amazônica: Mapeamento e análise descritiva de dados de desembarque no estado do Pará. In: XIV SIMPÓSIO BRASILEIRO DE SENSORIAMENTO REMOTO, 15., 2009, Natal. Anais XIV Simpósio Brasileiro de Sensoriamento Remoto, Natal, Brasil, 25-30 abril 2009, INPE, p. 4425-4432.

23. VALAVANIS, V. D. Geographic informaition systems in oceanography and fisheries. Taylor and Francis, New York. 2002.

24. WEST, B. Student attitude and the impact GIS on thinking skills and motivation. Journal of Geography.v.102, n.6, p.267-274. 2003.

25. YANEZ, R. E., CATASTI, V., BARBIERI, B.M.A. \& BOHM, G. S. Relationships between the small pelagic resources distribution and the sea surface temperatures recorded by NOAA satellites from Chile central zone. Investigaciones Marinas, 24: 107-122. 1996. 